\title{
Safety and feasibility of apheresis to harvest and concentrate parasites from subjects with induced blood stage Plasmodium vivax infection
}

\author{
Anand Odedra ${ }^{1,2^{*}}$ (D, Kari Mudie ${ }^{3}$, Glen Kennedy ${ }^{3}$, Rebecca E. Watts ${ }^{1}$, Emilie Rossignol ${ }^{1}$, Hayley Mitchell ${ }^{1}$, \\ Jeremy Gower', Maria Rebelo', Zuleima Pava', Rebecca Pawliw', Stephen Woolley ${ }^{1,2}$, David G. Lalloo², \\ Greg Robinson ${ }^{1}$, Sean Lynch ${ }^{1}$, Katharine A. Collins ${ }^{1,4}$, Fiona Amante ${ }^{1}$ and James McCarthy ${ }^{1}$
}

\begin{abstract}
Background: In the absence of a method to culture Plasmodium vivax, the only way to source parasites is ex vivo. This hampers many aspects of P. vivax research. This study aimed to assess the safety of apheresis, a method for selective removal of specific components of blood as a means of extracting and concentrating $P$. vivax parasites.

Methods: An iterative approach was employed across four non-immune healthy human subjects in single subject cohorts. All four subjects were inoculated with $\sim 564$ blood stage P. vivax (HMP013-Pv) and subjected to apheresis 10 to 11 days later. Blood samples collected during apheresis (haematocrit layers $0.5 \%$ to $11 \%$ ) were tested for the presence and concentration of P. vivax by microscopy, flow cytometry, $18 \mathrm{~S}$ rDNA qPCR for total parasites, and pvs 25 qRTPCR for female gametocyte transcripts. Safety was determined by monitoring adverse events. Malaria transmission to mosquitoes was assessed by membrane feeding assays.
\end{abstract}

Results: There were no serious adverse events and no significant safety concerns. Apheresis concentrated asexual parasites by up to 4.9-fold (range: 0.9-4.9-fold) and gametocytes by up to 1.45-fold (range: 0.38-1.45-fold) compared to pre-apheresis densities. No single haematocrit layer contained $>40 \%$ of all the recovered $P$. vivax asexual parasites. Ex vivo concentration of parasites by Percoll gradient centrifugation of whole blood achieved greater concentration of gametocytes than apheresis. Mosquito transmission was enhanced by up to fivefold in a single apheresis sample compared to pre-apheresis.

Conclusion: The modest level of parasite concentration suggests that the use of apheresis may not be an ideal method for harvesting P. vivax.

Trial Registration Australia New Zealand Clinical Trials Registry (ANZCTR) Trial ID: ACTRN12617001502325 registered on 19th October 2017. https://www.anzctr.org.au/Trial/Registration/TrialReview.aspx?id=373812.

Keywords: Malaria, Plasmodium, Apheresis, Parasite, Concentration

*Correspondence: anand_tony@hotmail.co.uk

${ }^{1}$ QIMR Berghofer Medical Research Institute, Herston Road, Brisbane, QLD 4006, Australia

Full list of author information is available at the end of the article

\section{Background}

Plasmodium falciparum is the most prevalent malaria parasite in Africa. However, Plasmodium vivax has a wider geographical distribution. In 2018, there were approximately 75 million cases of malaria due to $P$. vivax,

(c) The Author(s) 2021. This article is licensed under a Creative Commons Attribution 4.0 International License, which permits use, sharing, adaptation, distribution and reproduction in any medium or format, as long as you give appropriate credit to the original author(s) and the source, provide a link to the Creative Commons licence, and indicate if changes were made. The images or other third party material in this article are included in the article's Creative Commons licence, unless indicated otherwise in a credit line to the material. If material is not included in the article's Creative Commons licence and your intended use is not permitted by statutory regulation or exceeds the permitted use, you will need to obtain permission directly from the copyright holder. To view a copy of this licence, visit http://creativeco mmons.org/licenses/by/4.0/. The Creative Commons Public Domain Dedication waiver (http://creativecommons.org/publicdomain/ zero/1.0/) applies to the data made available in this article, unless otherwise stated in a credit line to the data. 
accounting for $50 \%$ of cases in South East Asia and 75\% of cases in the Americas [1].

In the absence of a method for continuous in vitro culture of $P$. vivax, parasites are usually sourced ex vivo from infected humans. This limits many aspects of the study of $P$. vivax research, including the development of interventions to control and eliminate $P$. vivax, such as diagnostics, drugs and vaccines. For example, a reliable source of P. vivax sporozoites is required to test and develop new drugs targeting the dormant liver-stage parasites-the hypnozoites. Currently this entails an expensive, logistically complex and unreliable process of sourcing $P$. vivaxinfected mosquitoes from endemic areas. In addition to the practical issues, parasites sourced in this way are not genetically homogenous. Thus, experiments are subject to possible effects of strain variability.

Experimental infection of human subjects with malaria termed volunteer infection studies (VIS) or controlled human malaria infection (CHMI) studies are increasingly being used for drug and vaccine development [2-7]. Infections can be induced by mosquito bite, injection of sporozoites or blood-stage parasites. The latter is used in the induced blood stage malaria (IBSM) model, where healthy subjects are injected with Plasmodium infected red blood cells. The IBSM model is being increasingly used for clinical studies of $P$. vivax [7-9].

It has recently been shown that $P$. vivax can be successfully transmitted from healthy subjects to Anopheles stephensi mosquitoes $[9,10]$. In this study sporozoites were harvested from the infected mosquitoes that were able to infect human hepatocytes in vitro [9]. Although this system offers the potential to study the biology of $P$. vivax malaria transmission and liver stage parasites, it is not a sustainable large-scale source of sporozoites for downstream work.

Apheresis is the removal of a specific component of an individual's blood. Currently, centrifugal apheresis is the preferred method whereby blood components are separated based on buoyancy. Computer-controlled automated apheresis systems undertake continuous removal, separation of the target component, and then return the remaining blood to the individual [11]. Automated erythrocytapheresis, also known as red cell exchange (RCE) has been used in the past for treatment of severe $P$. falciparum malaria with the rationale of reducing the parasitized red blood cell concentration by replacing Plasmodium-infected red blood cells with normal donor red blood cells [12]. However, the rapid parasite clearance resulting from artesunate therapy has negated the need for RCE as a treatment for severe malaria [13, 14].

Reported below are the results of a $P$. vivax clinical trial where apheresis was used as a means to harvest and concentrate all blood stages of $P$. vivax, including gametocytes, from human subjects experimentally infected with blood-stage $P$. vivax parasites.

\section{Methods \\ Study design}

The study presented here is a Phase 1 exploratory study that was conducted in four sequential single subject cohorts (ANZCTR Trial ID: ACTRN12617001502325) and performed at Q-Pharm Pty Ltd, Brisbane, Australia and the Apheresis Unit at the Royal Brisbane and Women's Hospital (RBWH), Australia between October 2017 and May 2019. The primary objective of the study was to determine the safety of the $P$. vivax infection in healthy subjects following inoculation with blood-stage parasites, and the safety of apheresis for collection of $P$. vivax parasites from experimentally infected subjects. Secondary objectives were to assess the feasibility of apheresis as a method of harvesting, concentrating and subsequently cryopreserving $P$. vivax parasites from healthy subjects. Exploratory objectives were to evaluate the potential for apheresis to be used as a method for producing a $P$. vivax human malaria parasite bank, to evaluate the transmission of $P$. vivax gametocytes to mosquitoes and to collect and store plasma and peripheral blood mononuclear cells harvested using apheresis.

Specific modifications to the study protocol, such as the apheresis procedure, were required between subjects in an attempt to optimize the procedure and meet the objectives. All changes made between subjects were based on the findings from previous subjects.

\section{Study subjects}

Healthy adult males and females, aged between 18 and 55 years who met all inclusion criteria and none of the exclusion criteria were eligible for participation. Subjects were required to be malaria-naïve, Duffy blood group positive and have blood type $\mathrm{O}$. Female subjects had to be $R h(D)$ positive. All subjects had to be available for a safety follow up period of three months. A full list of the inclusion/exclusion criteria for this study is included in the study protocol located in Additional file 1.

\section{Study conduct \\ Pre-clinical component}

A pre-clinical experiment was conducted prior to the clinical trial in order to confirm the feasibility of harvesting Plasmodium parasites using apheresis. The P. falciparum NF54 clone was used in these experiments [15] due to limited availability of $P$. vivax parasites. Plasmodium. falciparum infected red blood cells $(17.6 \mathrm{ml} ; 16 \mathrm{ml}$ blood with $0.1 \%$ asexual parasitaemia and $1.67 \mathrm{ml}$ blood with $0.01 \%$ gametocytaemia) were added to $450 \mathrm{ml}$ of fresh venous whole blood and subjected to ex vivo apheresis. 
Samples were collected from the $1 \%, 2 \%, 3 \%, 5 \%$ and $7 \%$ haematocrit (HCT) layers as determined by visualizing the colour saturation of the apheresis product. An automated haematology analyser (Sysmex XN-3000; Sysmex UK) was used retrospectively to confirm the HCT of samples collected during apheresis. Presence of parasites was assessed in each layer by $18 \mathrm{~S}$ qPCR [16] and microscopy.

\section{Clinical component}

Following intravenous injection of $P$. vivax (day 0), subjects were monitored by daily telephone calls until day 4 , when subjects visited the clinical unit daily until the day of apheresis. Subjects were monitored for adverse events (AEs), signs and symptoms of malaria infection, and blood was collected for 18S qPCR measurement of parasitaemia. The severity of AEs were determined by the common terminology of clinical trial adverse events (CTCAE) v. 4.03 [17].

The threshold for commencement of apheresis and treatment with artemether-lumefantrine was within $24 \mathrm{~h}$ of a parasitaemia $>20,000$ parasites $/ \mathrm{mL}$, or the Malaria Clinical Score reaching $>6$ [10], or at the Investigator's discretion. The morning that this threshold was reached (anticipated based on previous studies to be Day 10 or 11 [9], subjects were admitted to the clinical unit (Q-Pharm) for initial safety assessments before being escorted to the Apheresis Unit at RBWH by Q-Pharm staff. The Apheresis Unit is located in the Haematology Department at RBWH where patients are subject to donor or therapeutic apheresis. At the Apheresis Unit the subjects underwent the apheresis procedure as per the Standard Operating Procedure (Additional files 2, 3, 4 and 5) whilst being supervised by the apheresis specialist nurse and under the supervision of the responsible clinical haematologist (GK). The same apheresis nurse performed the apheresis procedure for all four subjects. The apheresis procedure lasted $1-4 \mathrm{~h}$. Subjects were then escorted back to the clinical unit and began treatment with artemether-lumefantrine (Riamet ${ }^{\circledR}$, Novartis Pharmaceuticals Australia Pty Ltd). Treatment consisted of six doses of 4 tablets at 12 hourly intervals (each tablet contains $20 \mathrm{mg}$ artemether and $120 \mathrm{mg}$ of lumefantrine). Subjects remained confined within the clinical unit for $48-72 \mathrm{~h}$ for safety monitoring. Following release from confinement, subjects attended protocol specified visits until three months post-treatment to monitor for signs of recrudescent parasitaemia and to assess late safety signals. Relapse is not a concern in the P. vivax IBSM studies as liver infection is bypassed and hypnozoites are not produced. A schematic of the study design is shown in Additional file 6: Fig. S1.

This study used an iterative adaptive design approach where subject safety and outcome data were analysed after each subject and modifications made to improve the chances of meeting the exploratory objectives in the subsequent subject. A summary of the changes instituted is shown in Table 1.

CMNC; continuous mononuclear cell collection, HCT; haematocrit. Protocols for subjects 1 to 4 and all experiments can be found in Additional files 7, 8, 9 and 10 . "Biological duplicates involved repeat $18 \mathrm{~S}$ qPCR testing from two separate blood samples from each HCT layer collected using apheresis.

When a HCT range is included the sample was taken from multiple HCT layers e.g. $5-7 \%=5 \%, 6 \%$ and $7 \%$ HCT. ${ }^{+}$Originally aimed to sample $8 \%$ HCT layer but actual sample consisted of $11 \%$ HCT. ${ }^{\#}$ During cohort 4

Table 1 Summary of main study design differences between subjects

\begin{tabular}{|c|c|c|c|c|}
\hline & Subject 1 & Subject 2 & Subject 3 & Subject $4^{\#}$ \\
\hline Apheresis procedure & CMNC & CMNC & CMNC & $\begin{array}{l}\text { Red cell depletion followed by CMNC } \\
\text { on red cell depletion product }\end{array}$ \\
\hline HCT layers sampled* & $1 \%, 2 \%, 3 \%, 5 \%, 7 \%$ & $1 \%, 2 \%, 3 \%, 5 \%, 7 \%$ & $\begin{array}{l}0.5 \%, 1 \%, 2 \%, 3 \% \\
5 \%, 7 \%, 11 \%+ \\
2-3 \%, 5-7 \% \\
1-7 \%\end{array}$ & $\begin{array}{l}\text { From the primary apheresis (HCT): } \\
\text { Intermediate }(64 \%) \\
\text { From the secondary apheresis (HCT): } \\
\text { Final }(3 \%) \text { Spare }(5 \%) \text { Waste }(42 \%)\end{array}$ \\
\hline Apheresis timepoint & 10 & 10 & $11 \mathrm{PM}$ & $11 \mathrm{AM}$ \\
\hline Mosquito feeding assay samples & $\begin{array}{l}\text { Pre-apheresis (with-Percoll } \\
\text { enrichment), 1\%, 2\%, 3\% HCT } \\
\text { layers }\end{array}$ & $\begin{array}{l}\text { Pre-apheresis (with- } \\
\text { Percoll enrich- } \\
\text { ment) }\end{array}$ & $\begin{array}{l}\text { Pre-apheresis } \\
\text { (with and } \\
\text { without-Percoll } \\
\text { enrichment) }\end{array}$ & $\begin{array}{l}\text { Pre-apheresis (without-Percoll enrich- } \\
\text { ment), Intermediate, Final, Waste }\end{array}$ \\
\hline $\begin{array}{l}\text { Whole blood:citrate ratio during } \\
\text { apheresis }\end{array}$ & $15: 1$ & $8: 1$ & $8: 1$ & $13: 1$ \\
\hline $\begin{array}{l}\text { Citrate added to apheresis collec- } \\
\text { tion bags }\end{array}$ & No & Yes & Yes & Yes \\
\hline Biological duplicates* & No & No & Yes & Yes \\
\hline
\end{tabular}


a red cell depletion was carried out, producing an intermediate bag sample, followed by a second apheresis procedure on the red cell depletion product. The second apheresis procedure involved sampling of $\sim 100 \mathrm{ml}$ of the lowest HCT layers of the sample (final bag) followed by $100 \mathrm{mls}$ of the subsequent lowest HCT layers (spare bag) and then the remainder $\sim 100 \mathrm{mls}$ (waste bag).

\section{Malaria challenge agent}

The $P$. vivax human malaria parasite (HMP) bank HMP013 was derived from blood group $\mathrm{O}$ rhesus positive blood donated from a returned traveller from India who presented with clinical manifestations of malaria [9]. The inoculum was prepared as previously described [18].

\section{Measurement of parasitaemia by qPCR}

Parasitaemia was quantified using $18 \mathrm{~S}$ qPCR targeting the highly conserved Plasmodium $18 \mathrm{~S}$ ribosomal RNA gene $[16,19]$. Quantitative reverse transcriptase PCR (qRT-PCR) assays were used to quantify gametocyte levels with assays targeting the $P$. falciparum pfs 25 (female) and $p f M G E T$ (male) gametocyte mRNA transcripts [20] and $P$. vivax pvs 25 (female) gametocyte mRNA transcripts [21].

\section{Flow cytometry}

Flow cytometry was performed to characterize cell populations present in samples collected during the apheresis process. A combination of stains and antibodies were used to identify cells containing DNA/RNA (SYBR Green I), white blood cells (WBCs) (CD45 antibody) and/or reticulocytes (CD71 antibody). Samples from subject 1 were stained with SYBR Green I (Molecular Probes); samples from subjects 2 and 3 were stained with SYBR Green I and CD45-PacificBlue; and samples from subject 4 were stained with SYBR Green I, CD45-Pacific Blue and/or CD71-APC. Samples were kept on ice or at $4-8{ }^{\circ} \mathrm{C}$ until analysed by flow cytometry.

\section{SYBR Green I staining}

A volume of $2.5 \mu \mathrm{l}$ or $1 \times 10^{6}$ cells from each sample was stained with $30-50 \mu \mathrm{l}$ of SYBR Green I at $10 \times$ for $30 \mathrm{~min}$ in the dark. After incubation, $200 \mu \mathrm{l}$ of FACS buffer (2\% fetal bovine serum in phosphate buffered saline) was added.

\section{Antibody staining}

Approximately $1 \times 10^{6}$ cells were stained with $5-10 \mu \mathrm{g} /$ $\mathrm{ml}$ of CD45-Pacific Blue or $2.5 \mu \mathrm{l}$ of CD71 stock solution for $30 \mathrm{~min}$ at $4-8{ }^{\circ} \mathrm{C}$ in the dark. Cells were washed twice with PBS by centrifugation at $1455 \times g$ for $4 \mathrm{~min}$, at $4{ }^{\circ} \mathrm{C}$. After the last wash $200 \mu \mathrm{l}$ of FACS buffer was added to the cells.
Double staining with SYBR Green I and CD45-Pacific Blue: A volume of $30 \mu \mathrm{l}$ of SYBR Green I at $10 \times$ was added to pelleted cells that were previously stained with CD45-Pacific Blue (as mentioned above) for $30 \mathrm{~min}$ at $4-8{ }^{\circ} \mathrm{C}$, in the dark. After incubation a volume of $200 \mu \mathrm{l}$ of FACS buffer was added.

Triple staining with SYBR Green I, CD45-Pacific Blue and CD71-APC: Approximately $1 \times 10^{6}$ cells were stained with $10 \mu \mathrm{g} / \mathrm{ml}$ of CD45-Pacific Blue, $2.5 \mu \mathrm{l}$ of CD71 stock solution and $30 \mu \mathrm{l}$ of SYBR Green I at 10x. Samples were incubated for $30 \mathrm{~min}$ in the fridge $\left(4-8{ }^{\circ} \mathrm{C}\right)$ in the dark. Cells were washed twice with PBS by centrifugation at $1455 \times g$ for $4 \mathrm{~min}$, at $4^{\circ} \mathrm{C}$. After incubation a volume of $200 \mu \mathrm{l}$ of FACS buffer was added.

\section{Flow cytometry analysis}

Samples from subjects 1,2 and 3 were acquired on a FACS CANTO II (BD Biosciences), using the $488 \mathrm{~nm}$ and $405 \mathrm{~nm}$ lasers. SYBR Green I positive cells were detected using a 530/30 nm band-pass filter and CD45-Pacific Blue positive cells were detected using a $450 / 50 \mathrm{~nm}$ band-pass filter. Samples from subject 4 were acquired on a LSR FORTESSA (BD Biosciences), using the $488 \mathrm{~nm}$, $640 \mathrm{~nm}$ and $405 \mathrm{~nm}$ lasers. SYBR Green I positive cells were detected using a 530/30 nm filter, CD45-Pacific Blue positive cells were detected using a $450 / 50 \mathrm{~nm}$ filter and CD71-APC positive cells were detected using a $670 / 14 \mathrm{~nm}$ filter. Flow cytometry data was analysed using Flow ${ }^{\circledR}{ }^{\circledR}$ software (version 10.8, Tree Star Inc, Oregon, USA).

\section{Microscopy}

Thick and thin smears were stained with Giemsa and examined under a $100 \times$ oil immersion objective by level 1 or 2 WHO certified malaria microscopists. Apheresis samples were expected to have a significantly different composition in terms of proportions of RBCs and WBCs when compared to whole blood (e.g. RBCs make up 1\% and approximately $46 \%$ of $1 \%$ HCT and whole blood samples respectively). As such, standard parasitaemia measures by microscopy were not feasible. It was decided that the expert microscopists would estimate parasitaemia based on sample composition.

\section{Mosquito feeding assays}

Transmissibility of pre-apheresis samples and postapheresis samples to An. stephensi was evaluated using membrane feeding assays (MFA) [9, 22]. For enriched MFA, gametocytes present in $80 \mathrm{~mL}$ of whole blood (pre-apheresis) were enriched in 70\% Percoll gradient. For direct MFA (DMFA), $650 \mu \mathrm{L}$ of pellet from whole blood (pre-apheresis) or from each apheresis sample was reconstituted to $50 \%$ haematocrit with malaria naïve 
$\mathrm{AB}+$ serum. Infection in midguts was assessed by qPCR [23] 8 days after the feeding assays. For logistic reasons, enriched MFA was not carried out in subject 4. Following consideration of gametocyte levels measured by qRT-PCR targeting pvs25, DMFA was not carried out in subject 2 .

\section{Apheresis procedures}

Apheresis was carried out using a Spectra Optia v11.3 apheresis system (Terumo BCT, Inc Tokyo Japan) as detailed in the Additional file 11.

The continuous mononuclear cell collection procedure was used to sample from the targeted HCT layers from the blood of subjects 1 to 3 . The targeted HCT layers that were sampled from these subjects ranged from the platelet rich layer through to the red cell rich layer.

A double stage procedure was used to collect the targeted HCT layers from subject 4 . In the first stage, a red cell depletion procedure was used to collect approximately $500 \mathrm{ml}$ of packed red blood cells from the subject. Targeted HCT layers were then collected from the red cell concentrate using the polymorphonuclear (PMN) collection procedure. The starting product (red cell concentrate) for the second stage of this procedure had a significantly higher HCT than the whole blood of subjects 1 to 3 and sampling focussed on the higher HCT layers. This was the rationale for using a PMN collection in subject 4 rather than the CMNC collection used in subjects 1 to 3 .

\section{Statistical analysis}

Continuous data was summarized using descriptive statistics (mean and standard deviation, or median and interquartile range). Categorical data was presented using $\mathrm{N}$ and \%. Descriptive statistics were produced using Microsoft Excel ${ }^{\circledR}$ (version 1903). GraphPad ${ }^{\circledR}$ Prism was used for the construction of all figures.

\section{Results}

\section{Pre-clinical experiment}

The feasibility of extracting Plasmodium parasites from blood using apheresis was initially assessed using cultured P. falciparum parasites. The 1\% HCT layer contained the greatest concentration of all parasites as determined by $18 \mathrm{~S}$ qPCR, with a 1.3 -fold concentration of all parasites and a 3.7 and eightfold concentration for female and male gametocytes, compared to pre-apheresis (Additional file 6: Fig. S5 and Tables 1, 2 and 3). The $2 \%$ HCT layer contained the greatest concentration of asexual parasites by microscopy (2.7-fold concentration; Additional file 6: Fig. S6A and Table S4). The highest concentration of gametocytes detected by microscopy was seen in samples collected from the 1\% HCT layer (76fold; Additional file 6: Fig. S6A and Table S4) with stage 1 gametocytes making up the largest proportion (37.5\%). All stages of gametocytes apart from stage 5 gametocytes were visualized. Both asexual parasites and gametocytes were also visualized by microscopy in the $1 \%, 3 \%$ and 5\% HCT layers (Additional file 6: Fig. S6B and Table S5). These results demonstrated the technical feasibility of the

Table 2 Summary of the main safety findings

\begin{tabular}{|c|c|c|c|c|c|}
\hline & Subject 1 & Subject 2 & Subject 3 & Subject 4 & Total AEs \\
\hline SAES & 0 & 0 & 0 & 0 & 0 \\
\hline AEs & 20 & 13 & 15 & 20 & 68 \\
\hline AEs related to malaria & 13 & 8 & 14 & 19 & 54 \\
\hline AEs related to apheresis & 2 & 3 & 0 & 4 & 9 \\
\hline Max temp ${ }^{\circ} \mathrm{C}$ & 40.2 & 38.8 & 40.2 & 39.6 & $\mathrm{~N} / \mathrm{A}$ \\
\hline Max malaria clinical score & 8 & 1 & 2 & 7 & $\mathrm{~N} / \mathrm{A}$ \\
\hline Ibuprofen use & $400 \mathrm{mg} \times 5$ & $400 \mathrm{mg} \times 5$ & $400 \mathrm{mg} \times 5$ & nil & $\mathrm{N} / \mathrm{A}$ \\
\hline Acetaminophen use & $1 \mathrm{~g} \times 4 ; 500 \mathrm{mg} \times 1$ & $1 \mathrm{~g} \times 2$ & $1 \mathrm{~g} \times 4$ & $500 \mathrm{mg} \times 2 ; 1 \mathrm{~g} \times 2$ & $\mathrm{~N} / \mathrm{A}$ \\
\hline Peak ALT (IU/L) & 111 & 118 & 47 & 80 & N/A \\
\hline Peak AST (IU/L) & 83 & 57 & 42 & 44 & $\mathrm{~N} / \mathrm{A}$ \\
\hline Platelet Nadir (× 109/L) & 119 & 98 & 99 & 75 & N/A \\
\hline $\begin{array}{l}\text { Maximum drop in haemoglobin from } \\
\text { baseline }(\mathrm{g} / \mathrm{L})\end{array}$ & 17 & 9 & 25 & 20 & $\mathrm{~N} / \mathrm{A}$ \\
\hline Lymphocyte Nadir (× 109/L) & 0.33 & 0.43 & 0.58 & 0.75 & N/A \\
\hline Peak parasitaemia (parasites/mL) & 15,943 & 35,156 & 64,243 & 44,431 & N/A \\
\hline
\end{tabular}

$S A E$ serious adverse event, $A E$ adverse event

Summary of the main safety findings encountered during the study 


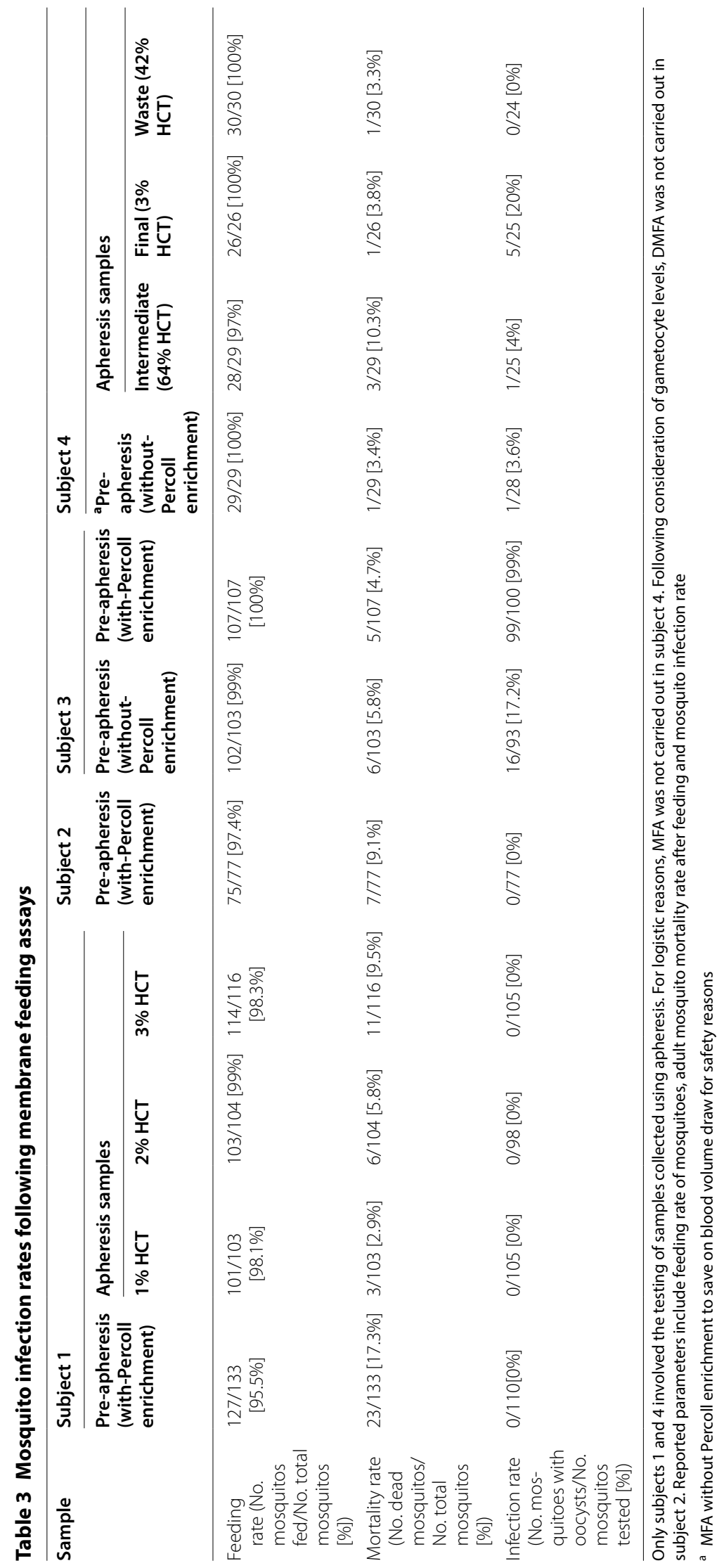


approach and the experiment was allowed to proceed to the clinical stage.

\section{Clinical experiment}

The course of $P$. vivax infection (Fig. 1) followed the same course as demonstrated in previous studies [9]. Subjects 1 and 2 were treated with artemether/lumefantrine 10 days post malaria inoculation. To augment pre-apheresis parasitaemia, treatment of subjects 3 and 4 was delayed to day 11. Apheresis could be delayed safely as the clinical signs observed for subjects 3 and 4 were mild enough for the artemether/lumefantrine treatment, only administered after apheresis, to be postponed by $24 \mathrm{~h}$. All subjects became 18S qPCR negative for parasites within $72 \mathrm{~h}$ of treatment initiation.

A summary of the key differences in the planning of the clinical trial in each of the four subject cohorts is shown in Table 1. During cohort 4 a red cell depletion was carried out, producing an intermediate bag sample, followed by a second apheresis procedure on the red cell depletion product. The second apheresis procedure involved sampling of $\sim 100 \mathrm{ml}$ of the lowest HCT layers of the sample (final bag) followed by $100 \mathrm{mls}$ of the subsequent lowest HCT layers (spare bag) and then the remainder (waste bag) (Additional file 6: Fig. S3). A schematic of the sampling that occurred during cohort 4 can be seen in Additional file 6: Fig. S3.

\section{Safety}

A total of 68 AEs occurred in the 4 subjects (Table 2). No Serious AEs were reported. The majority of AEs were mild or moderate. Five severe AEs occurred in 4 subjects: one episode of neutropenia $\left(0.68 \times 10^{9} / \mathrm{L}\right.$ [0.45 $\left.\times \mathrm{LLN}\right]$; duration 8 days), two of lymphopenia $\left(0.43 \times 10^{9} / \mathrm{L}\right.$ $[0.43 \times \mathrm{LLN}]$ and $0.33 \times 10^{9} / \mathrm{L}[0.33 \times \mathrm{LLN}]$; both lasting 3 days), and two of fever (both $40.2^{\circ} \mathrm{C}$; duration $30 \mathrm{~min}$ and $25 \mathrm{~min}$ ). All severe AEs were transient and resolved by the end of the study. The majority of AEs (54/68; $79.4 \%)$ were attributed to malaria, while $9 / 68$ (13.2\%) were attributed to apheresis. These included neutropenia (one subject nadir $0.68 \times 10^{9} / \mathrm{L}$ ) which was recorded three times due to changes in severity, two cases of lymphopenia (nadir $0.33 \times 10^{9} / \mathrm{L}$ and $0.75 \times 10^{9} / \mathrm{L}$ ) and two cases of leukopenia (nadir $2.2 \times 10^{9} / \mathrm{L}$ and $2.4 \times 10^{9} / \mathrm{L}$ ). One subject had an episode of herpes labialis (herpes simplex virus-1 PCR positive). One subject experienced mild hypophosphatemia $(0.70 \mathrm{mmol} / \mathrm{L})$ of two days duration. All AEs attributed to apheresis apart from the case of hypophosphataemia were considered to be possibly related to apheresis, malaria or a combination of both.

\section{Characteristics of samples collected by apheresis}

The red blood cell counts in samples from the various HCT layers were generally in alignment with what would be expected (Additional file 6: Table S6), except in two

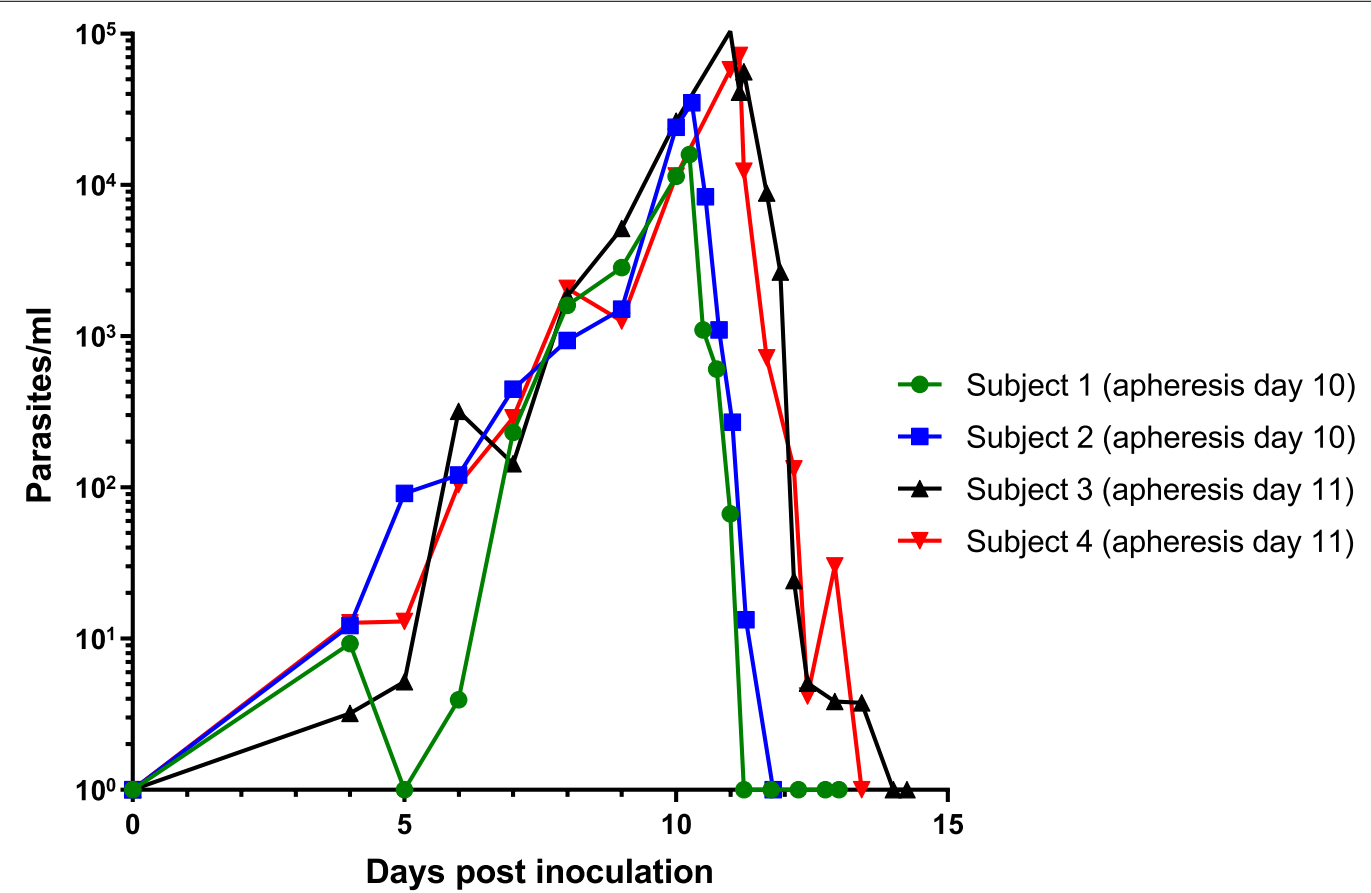

Fig. 1 Parasite growth curves. Parasitaemia as measured by $18 \mathrm{~S}$ qPCR in all four subjects. Day 0 represents the day of inoculation. Apheresis occurred on day 10 for subjects 1 and 2, and day 11 for subjects 3 and 4 
subjects. In subject 2 the red blood cell counts in samples from the $2 \%$ HCT layer was closer to what would be expected from a 3\% HCT layer and vice versa, and the sample from the $8 \%$ HCT sample in subject $3 \mathrm{had}$ a HCT of $11 \%$. The variability in expected HCT and actual HCT is a potential limitation and should be considered when designing any future studies. The cell composition of samples collected using apheresis in subjects 1 to 3 (Additional file 6: Fig. S7A) showed an $\sim 60$ to 170fold decrease in the $\mathrm{RBC}: \mathrm{WBC}$ ratio from pre-apheresis samples compared to apheresis samples. Among samples collected by apheresis from subject 4 , where a double apheresis process was undertaken, the $\mathrm{RBC}$ :WBC ratio was close to that of the pre-apheresis sample (Additional file 6: Fig. S7B), with the exception of the final bag sample ( $3 \%$ HCT). Reticulocyte counts measured on the Sysmex analyser were the highest in subject 2: $0.23 \times 10^{9} / \mathrm{L}$ (reference range for whole blood: $25-120 \times 10^{9} / \mathrm{L}$ ).

\section{Concentration of asexual parasites}

$18 \mathrm{~S}$ qPCR targets the highly conserved plasmodium $18 \mathrm{~S}$ ribosomal RNA gene present in asexual parasites and gametocytes $[14,18]$. However, based on the $P$. vivax life cycle, microscopy and $P$. vivax female gametocyte qRT PCR data (pvs25) it was determined that the vast majority of parasites, detected using $18 \mathrm{~S}$ qPCR, were asexual parasites.

No single HCT layer contained $>40 \%$ of all the recovered $P$. vivax asexual parasites (Fig. 2). An increase in parasite concentration per $\mathrm{ml}$ of sample attained via apheresis occurred as HCT increased in subjects 1 to 3
(Fig. 2a), with some variation in relative enrichment of parasites in apheresis samples compared to pre-apheresis samples at any given HCT (Fig. 2a). The highest concentration achieved was a 4.9-fold increase in parasite density in the 7\% HCT layer in subject 1 (Fig. 2a and Additional file 6: Table S7). There was no apparent enrichment of parasites when the procedure was modified to include a second apheresis process (subject 4; Fig. 2b).

When parasite concentration was adjusted for RBC count, all apheresis samples collected from subjects 1 to 3 demonstrated enrichment for asexual parasites compared to pre-apheresis (Additional file 6: Fig. S2A). In general, when parasite counts were corrected for RBC count, parasite enrichment was highest in the samples collected at the lower HCT (Additional file 6: Fig. S2 and Table S7). The relative concentration of parasites from subject 4 , where the second apheresis procedure was performed, was observed in the lowest HCT samples. In particular, the final bag (3\% HCT) and the spare bag (5\% HCT) samples had relative enrichment levels of 20- and 8-fold respectively compared to pre-apheresis (Additional file 6: Fig. S2 and Table S7).

\section{Concentration of $P$. vivax gametocytes}

Analysis of the apheresis samples from subject 4, where the double apheresis process was undertaken, demonstrated an increase in the level of female gametocytes of 1.45 -fold per $\mathrm{ml}$ in the apheresis sample compared to a venous blood sample collected pre-apheresis, as

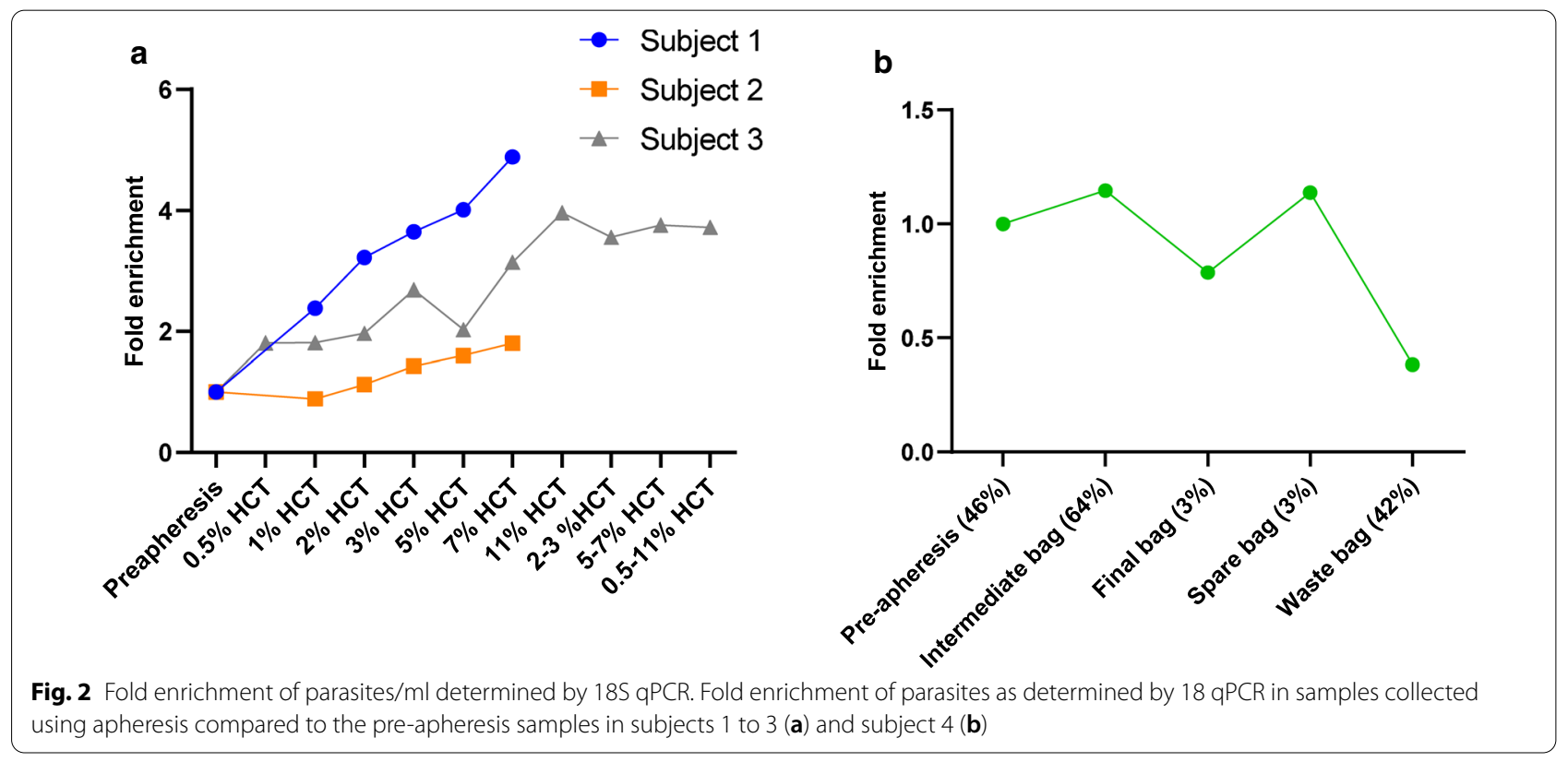


determined by qRT PCR for the gametocyte-specific transcript pvs25 (Fig. 3b and Additional file 6: Table S8).

Subjects 1, 2 and 3 demonstrated a reduction in the level of female gametocytes compared to pre-apheresis (Fig. 3a and Additional file 6: Table S8). In most cases the reduction was $>10$-fold. Percoll concentration of whole blood taken pre-apheresis resulted in a significant enrichment (up to 45 -fold in subject 3) of pvs 25 compared to pre-apheresis samples not enriched with Percoll (Fig. 3c and Additional file 6: Table S8).

In subject 1 the Percoll enrichment experiment failed due to technical issues resulting from suboptimal processing of the sample.
When gametocyte concentrations were corrected for red blood cell counts, enrichment levels were generally higher in the lower HCT samples (Additional file 6: Fig. S4a, b and Table S8). Relative enrichment of gametocytes in subjects 1, 2 and 3 was lower than for total parasites, with a maximum enrichment of 6.2 -fold in the $0.5 \% \mathrm{HCT}$ layer (Additional file 6: Table S8). In subjects 3 and 4 enrichment relative to red blood cell count was observed (Additional file 6: Fig. S4A, B). In subject 4, the greatest enrichment of gametocyte transcripts in the double apheresis process was observed in the spare bag $(5 \%$ HCT) with an enrichment of 6.1-fold (Additional file 6: Fig. S4A, B and Table S8).

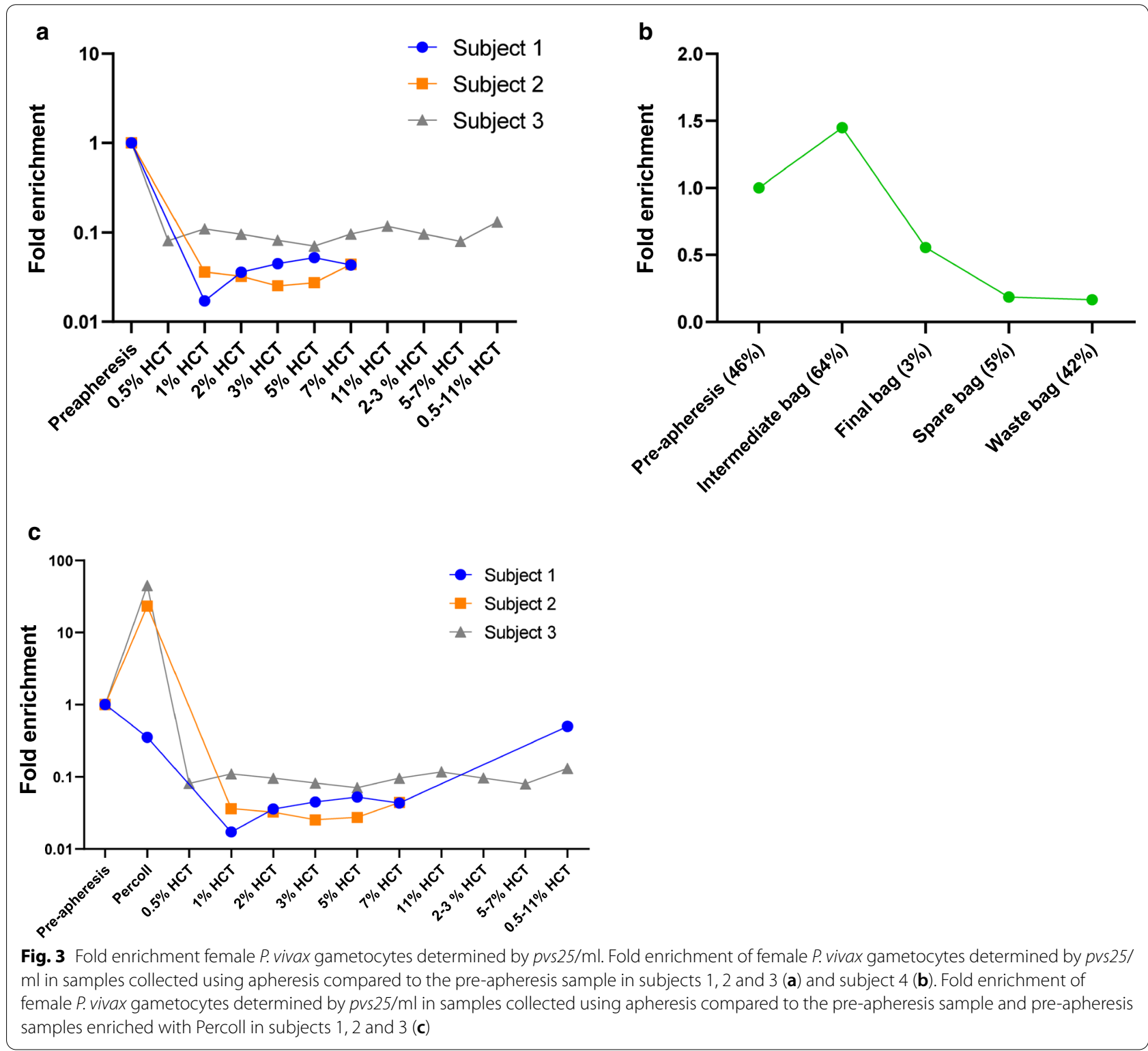




\section{Flow cytometry}

In subjects 2 and 3, it was observed that as sampling was undertaken from progressively lower haematocrits there was an increase in the percentage of WBCs (Additional file 6: Figs. S10 and S11). A small percentage of SYBR Green I+ and CD45- cells, which could represent either parasitized RBCs or reticulocytes, were detected in samples from subjects 2 and 3 (Fig. 4a, b); pooled samples collected at 2-3\% HCT from subject 3 had the highest concentration $(0.59 \%)$ of SYBR Green I+ and CD45-cells (Fig. 4b). In subject 4, where CD71 antibody staining was used to identify reticulocytes, the highest levels of reticulocytes (CD71 + cells) were observed in the final bag (3\% HCT) and the spare bag (5\% HCT) (Fig. 4c). Microscopic analysis of these samples suggested that the reticulocyte population detected by flow cytometry consisted of uninfected reticulocytes. No parasitized RBCs or reticulocytes could be detected by flow cytometry, and thus indicating that these cells are absent or below the limit of detection by flow cytometry.

\section{Microscopy}

The high concentration of WBCs in apheresis samples, meant many of the thick and thin blood smears were extremely difficult to read (Additional file 6: Tables S9 and S10). Among samples where it was possible to read the films, parasite counts were low, and in alignment with the counts obtained by qPCR (Additional file 6:

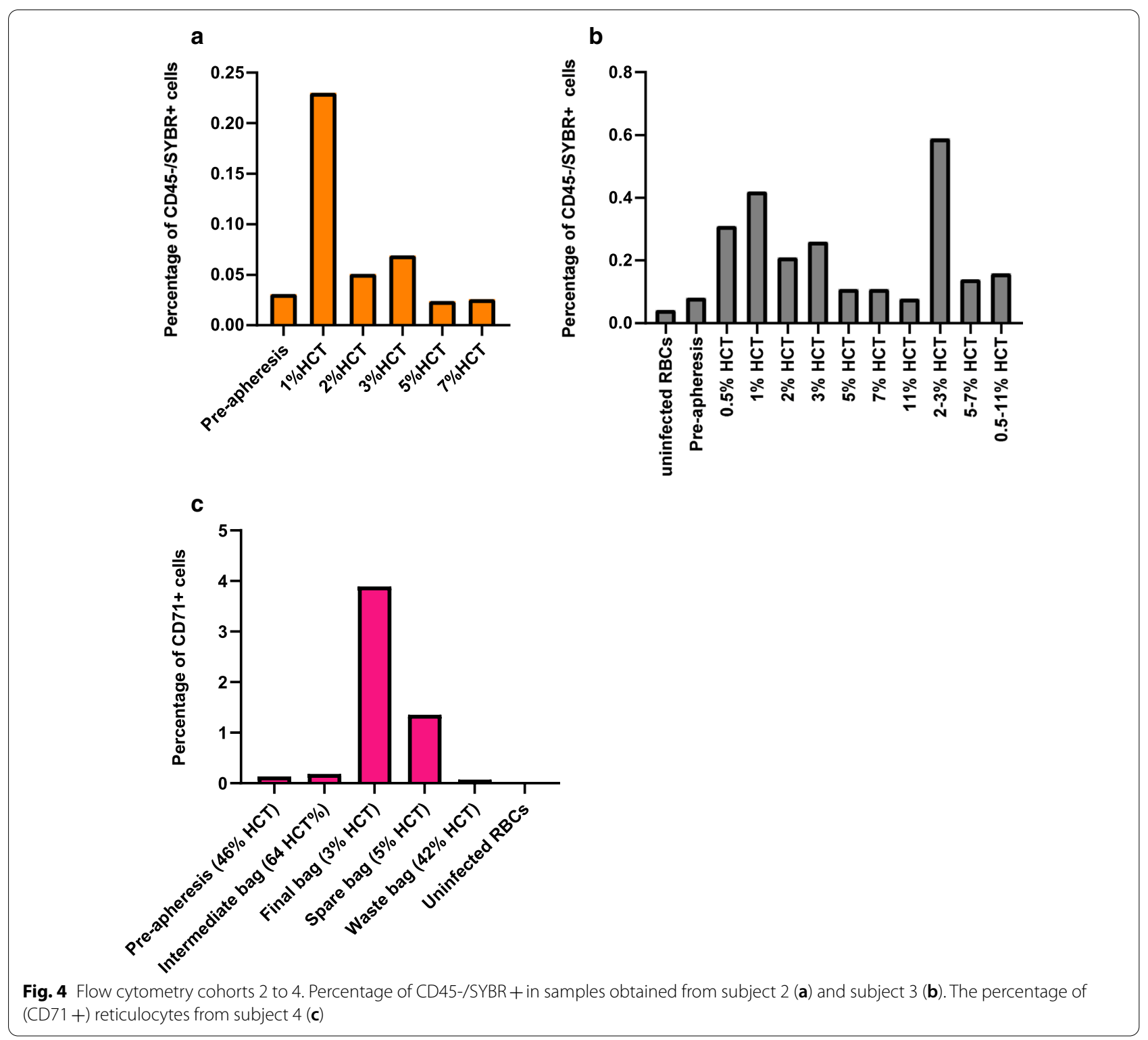


Tables S9 and S10). Notwithstanding the technical difficulties in reading the slides and the low parasite counts, no apparent concentration of parasites was observed in any of the apheresis samples compared to pre-apheresis. The 3\% HCT sample in subject 3 contained 7 parasitized cells compared to 0 in the pre-apheresis sample and the $1 \%$ HCT sample in subject 1 demonstrated a fourfold increase in the number of visualized parasites compared to pre-apheresis. In general, higher parasite numbers were seen in the lower HCT layers. The vast majority of parasitized RBCs contained ring form parasites, with trophozoites and gametocytes observed in samples from subjects 3 and 4 only (Additional file 6: Tables S9 and S10).

\section{Mosquito transmission}

Membrane feeding of pre-apheresis or post Percoll enrichment samples were undertaken in all 4 subjects, but for logistical reasons membrane feeding on apheresis samples could only be undertaken in subjects 1 and 4 (Table 3).

None of the samples from subject 1 or 2 resulted in successful mosquito transmission (Table 3 ). In subject 3 , the infection rate from the Percoll-enriched sample was 5.8-fold higher than the infection rate from the nonenriched pre-apheresis sample ( $99 \%$ vs $17.2 \%$ [Table 3]). Likewise, in subject 4 , the infection rate from the final bag $(3 \% \mathrm{HCT})$ was 5.5 -fold higher than the infection rate from either the pre-apheresis sample or the intermediate bag $(64 \% \mathrm{HCT})$ (pre-apheresis: 3.6\%, intermediate bag: $4 \%$, final bag: $20 \%$ [Table 3]).

The final sample bag $(3 \% \mathrm{HCT})$ in subject 4 was the only sample obtained using apheresis that demonstrated an increase in transmission over samples obtained preapheresis. Due to logistic issues the spare bag $(5 \% \mathrm{HCT})$, which contained the greatest enrichment for gametocytes compared to pre-apheresis when adjusted for red cell counts in subject 4 (Additional file 6: Fig. S4b) was not subject to membrane feeding.

\section{Additional 185 qPCR testing}

To investigate for possible accumulation of parasites in the magnets and tubing structures in the apheresis cassette, qPCR testing was carried out on three blood clots with diameters of up to $1 \mathrm{~cm}$ found near two magnets, and a silo like structure that formed part of the single use apheresis tubing and processing cassette from subject 3 (Additional file 6: Fig. S13). Clots were thoroughly homogenized and tested by $18 \mathrm{~S}$ qPCR. The greatest enrichment observed relative to pre-apheresis whole blood was 1.4-fold in the apheresis cassette magnet 2 sample (Additional file 6: Fig. S14 and Table S11).
To investigate whether haemolysis may be taking place in the apheresed blood resulting in release of parasite DNA into the extracellular fluid, plasma from apheresis samples in subject 3 was collected by centrifugation of the sample, with the plasma subject to $18 \mathrm{~S}$ qPCR testing. However, no significant accumulation of parasite DNA was detected in the plasma compared to pre-apheresis (Additional file 6: Fig. S14 and Table S11).

\section{Discussion}

Using apheresis it was possible to achieve modest concentration of both asexual and gametocyte stages of $P$. vivax. However, the modest level of parasite enrichment (4.9-fold and 1.45-fold for asexual parasites and gametocytes respectively) was deemed to be insufficient for downstream research. Furthermore the relatively low parasite levels, particularly of gametocytes, meant that the figures may be subject to chance variations in parasite levels.

Results of this study suggest that apheresis in healthy subjects infected with blood-stage $P$. vivax parasites is safe. No serious adverse events were encountered, with all adverse events having resolved by the end of study. The majority of adverse events were malaria related, and in line with previous $P$. vivax IBSM studies [7, 8]. Adverse events related to apheresis consisted largely of asymptomatic transient reductions in haematology parameters.

After correction for red blood cell number, parasite quantitation by qPCR suggested that both asexual parasites and gametocytes were preferentially concentrated in the lower HCT layers (Additional file 6: Figs. S2 and S4). Furthermore, the pre-clinical experiment demonstrated a selective concentration of P. falciparum gametocytes compared to asexual parasites in lower HCT layers (Additional file 6: Fig. S6A and Table S4). However as the pre-clinical experiment did not involve collection of blood from an infected subject they do not fully replicate those collected ex vivo, for example being subject to host interactions such as sequestration, thus limiting the utility of this work in predicting what would be the situation in natural infection. Findings from the pre-clinical and clinical experiments were consistent with the previously published observations of the buoyancy of Plasmodium parasites [24].

Several scenarios were considered to explain why concentration of parasites using apheresis was lower than expected. Firstly, as gametocyte concentrations in apheresis samples were around the level of detection of the $p v s 25$ by qRT-PCR, minor variation in concentration may have been difficult to quantify [25]. Secondly, infected RBCs containing magnetic haemozoin [26] may have attached to ferromagnetic components of the apheresis apparatus. Thirdly, it is possible that lysis of asexual 
parasites and gametocytes occurred during the apheresis procedure. This latter hypothesis is supported by a recent report suggesting that parasite maturation results in increasing fragility of $P$. vivax infected red blood cells [27]. Although it may have been possible to assess for low level haemolysis during the procedure, for example by measuring haptoglobin levels, controlling for a range of other variables would have been difficult.

An enhanced level of transmission to mosquitoes compared to whole blood samples collected pre-apheresis was only observed on one occasion (final sample bag [3\% $\mathrm{HCT}$ ] in subject 4), corresponding to the higher gametocyte concentration in this sample compared to the pre-apheresis whole blood (Fig. 4b). A possible explanation of the low success in the transmission studies was the difficulty in maintaining tight temperature control to prevent exflagellation of male gametocytes [28], thereby negatively impacting gametocyte infectivity [29]. Blood was most vulnerable to a temperature drop whilst in the apheresis equipment itself. It was not possible to heat apheresis equipment, and it was deemed impractical to heat the room where apheresis took place to $>35$ ${ }^{\circ} \mathrm{C}$. Temperature monitoring was not possible during the experiments. However, the demonstration of transmission success in the final bag $(3 \% \mathrm{HCT})$ indicates that at least some gametocytes were maintained within a temperature range that did not trigger exflagellation. Regardless of the underlying cause, recent reports of success in improving concentration of gametocytes and enhanced transmission by either Percoll $[9,10]$ or magnetic bead [30] enrichment suggests that such methods are superior for concentration of gametocytes for mosquito transmission experiments.

Each of the vials from the HMP bank used in this study to infect subjects contain $2.08 \times 10^{6}$ parasites. Based on the greatest level of asexual enrichment per $\mathrm{ml}$ of sample observed (7\% HCT, subject 1), calculations suggest that apheresis alone can create parasites vials with a maximum of $7.92 \times 10^{4}$ parasites (Additional file 6). Therefore, to create a HMP bank using an apheresis approach equivalent to the one used to infect volunteers in this study a $>25$-fold increase in pre-apheresis parasitaemia would be required, equating to $>650,000$ parasites $/ \mathrm{ml}$. Attaining such high parasitaemia would likely lead to significant discomfort in study volunteers, and therefore raise significant ethical concerns. Therefore, unless significant improvements in enrichment can be attained, apheresis should not be used to create HMP banks, and the current practice of collecting blood by venesection is preferable.

Strengths of the study include the wide sampling across HCT layers (1\%, $2 \%, 3 \%, 5 \%$ and $7 \%$ ) and the use of multiple methods to enumerate parasites (flow cytometry, microscopy and qPCR). Although only a small number of subjects were studied using this approach, the lack of promising data meant that continuation of the trial was deemed inappropriate by the safety review team and the study was terminated.

\section{Conclusion}

Given the moderate levels of enrichment and the significant ethical, financial and logistical concerns surrounding P. vivax IBSM studies, it was decided that further apheresis studies are not warranted at this point. If apheresis were to be used again in the context of Malaria VIS, it should ideally only be carried out in subjects with normal haematological parameters at screening to avoid potential complications or the need for blood transfusion.

\section{Supplementary Information}

The online version contains supplementary material available at https://doi. org/10.1186/s12936-021-03581-w.

Additional file 1. Apheresis of subjects with induced blood stage $p$. vivax protocol.

Additional file 2. Apheresis Cohort 1 standard operating procedure. Additional file 3. Apheresis Cohort 2 standard operating procedure. Additional file 4. Apheresis Cohort 3 standard operating procedure. Additional file 5. Apheresis Cohort 4 standard operating procedure. Additional file 6. Supplementary Material v1.0.

Additional file 7. Apheresis Cohort 1 laboratory standard operating procedure.

Additional file 8. Apheresis Cohort 2 laboratory standard operating procedure.

Additional file 9. Apheresis Cohort 3 laboratory standard operating procedure.

Additional file 10. Apheresis Cohort 4 laboratory standard operating procedure.

Additional file 11. Spectra Optia Brochur System Overview.

\section{Abbreviations}

VIS: Volunteer infection studies; CHMI: Controlled human malaria infection; IBSM: Induced blood stage malaria; RCE: Red cell exchange; QIMR: Queensland Institute of Medical Research; RBWH: Royal Brisbane and Women's Hospital; HREC: Human Research Ethics Committee; GCP: Good Clinical Practice; HCT: Haematocrit; CTCAE: Common terminology of clinical trial adverse events; CMNC: Continuous mononuclear cell collection; PCR: Polymerase chain reaction; qPCR: Quantitative polymerase chain reaction; qRT PCR: Quantitative reverse transcriptase polymerase chain reaction; WBCs: White blood cells; RBCs: Red blood cells; MFA: Membrane feeding assays; DMFA: Direct membrane feeding assays; PMN: Polymorphonuclear; LLN: Lower limit of normal; ULN: Upper limit of normal; SAE: Serious adverse event; AE: Adverse event.

\section{Acknowledgements}

Indera Govender provided technical advice regarding the apheresis procedure, Adam Potter assisted with protocol PICF and manuscript preparation, Helen Jennings provided technical advice on safety and quality control of blood products produced during the study, Katherine Trenholme provided assistance and technical advice during laboratory experiments, Renee Atkinson provided clinical nursing supervision to volunteers and oversaw data collection, Sue Mathison provided clinical nursing supervision to volunteers and oversaw data collection, Matthew Adams provided assistance and technical advice during laboratory experiments, LTCOL Ken Lilley provided microscopy 
input, LTCOL Ivor Harris provided microscopy input, CAPT Jo Kizu provided microscopy input, Stephan Chalon acted as an independent medical monitor, Dennis Shanks acted as an independent medical monitor.

\section{Authors' contributions}

AO oversaw study conception, design of the study, study conduct, data collection, data analysis and manuscript production. KM oversaw the apheresis procedure for each subject and involved in study conception, design of the study, study conduct, data collection, data analysis and approval of the manuscript. GL provided clinical oversight for the apheresis procedure for each study subject and was involved in study conception, design of the study, data analysis and approval of the manuscript. REW was involved in study conception, design of the study including the production of the study protocol and patient information and consent form. EM was involved in design of the study, study conduct, data collection, data analysis and approval of the manuscript. HM was involved in design of the study, study conduct, data collection, data analysis and approval of the manuscript. JG was involved in design of the study, study conduct, data collection, data analysis and approval of the manuscript. MR was involved in design of the study, study conduct, data collection, data analysis and approval of the manuscript. ZP was involved in design of the study, study conduct, data collection, data analysis and approval of the manuscript. RP was involved in design of the study, study conduct, data collection, data analysis. SW was involved study conduct, data collection and approval of the manuscript. DGL was involved in design of the study, data analysis and approval of the manuscript. GR study conduct and data collection. KAC was involved in design of the study, study conduct, data collection, data analysis and approval of the manuscript. FA was involved in design of the study, study conduct, data collection, data analysis and approval of the manuscript. JMC provided senior scientific input and clinical oversight on study conception, design of the study, study conduct, data analysis and manuscript production. All authors read and approved the final manuscript.

\section{Funding}

This work was funded by a National Health and Medical Research Council (NHMRC) of Australia Program grant (1132975) awarded to JSM. JSM was supported by a NHMRC Practitioner Fellowship.

\section{Availability of data and materials}

Data collected for this study will be made available immediately after article publication with no end date. De-identified datasets containing the variables analysed for the primary and secondary objectives will be made available as well as other supporting documents (e.g., protocol and informed consent). Investigators who seek access to individual subject data will contact the corresponding author $(\mathrm{AO})$ to receive instructions on the formal request process, which will include the submission of a brief proposal. The proposal will be reviewed for merit and feasibility by the corresponding author (AO). Investigators will be notified of the decision within 30 days of receipt. If the request is accepted, a data transfer agreement covering relevant conditions will be required.

\section{Ethics approval and consent to participate}

The study was conducted in accordance with the protocol approved by the QIMR Berghofer Human Research Ethics Committee (HREC) and the RBWH HREC, the principles of the Declaration of Helsinki (recommendations guiding medical doctors in biomedical research involving human subjects, Fortaleza, Brazil 2013), the NHMRC National Statement on Ethical Conduct in Human Research (2007), and the Notes for Guidance on Good Clinical Practice (GCP) (CPMP/ICH/135/95) as adopted by the Australian Therapeutic Goods Administration (2000). All amendments and addenda to the protocol were similarly submitted to the QIMR Berghofer HREC and the RBWH HREC for approval prior to their implementation. All subjects gave written informed consent before being included in the study.

\section{Consent for publication}

All subjects gave written informed consent for their anonymized data to be published.

\section{Competing interests}

The authors declare that they have no competing interests.

\section{Author details}

1 QIMR Berghofer Medical Research Institute, Herston Road, Brisbane, QLD 4006, Australia. ${ }^{2}$ Liverpool School of Tropical Medicine, Pembroke Place, Liverpool L3 5QA, UK. ${ }^{3}$ Haematology Department, Royal Brisbane and Women's Hospital, Butterfield Street, Herston, Brisbane, QLD 4029, Australia. ${ }^{4}$ Department of Medical Microbiology, Radboud University Medical Centre, Geert Grooteplein Zuid 10, 6525 GA Nijmegen, The Netherlands.

\section{Received: 31 March 2020 Accepted: 4 January 2021}

Published online: 14 January 2021

\section{References}

1. WHO. World Malaria Report 2019. Geneva, World Health Organization; 2019.

2. Tran TM, Jones MB, Ongoiba A, Bijker EM, Schats R, Venepally P, et al. Transcriptomic evidence for modulation of host inflammatory responses during febrile Plasmodium falciparum malaria. Sci Rep. 2016;6:31291.

3. McCall MB, Netea MG, Hermsen CC, Jansen T, Jacobs L, Golenbock D, et al. Plasmodium falciparum infection causes proinflammatory priming of human TLR responses. J Immunol. 2007;179:162-71.

4. Sauerwein RW, Roestenberg M, Moorthy VS. Experimental human challenge infections can accelerate clinical malaria vaccine development. Nat Rev Immunol. 2011;11:57-64.

5. Sanderson F, Andrews L, Douglas AD, Hunt-Cooke A, Bejon P, Hill AV. Blood-stage challenge for malaria vaccine efficacy trials: a pilot study with discussion of safety and potential value. Am J Trop Med Hyg. 2008;78:878-83.

6. McCarthy JS, Sekuloski S, Griffin PM, Elliott S, Douglas N, Peatey C, et al. A pilot randomised trial of induced blood-stage Plasmodium falciparum infections in healthy volunteers for testing efficacy of new antimalarial drugs. PLoS ONE. 2011;6:e21914.

7. Griffin P, Pasay C, Elliott S, Sekuloski S, Sikulu M, Hugo L, et al. Safety and reproducibility of a clinical trial system using induced blood stage Plasmodium vivax infection and its potential as a model to evaluate malaria transmission. PLoS Negl Trop Dis. 2016;10:e0005139.

8. McCarthy JS, Griffin PM, Sekuloski S, Bright AT, Rockett R, Looke D, et al. Experimentally induced blood-stage Plasmodium vivax infection in healthy volunteers. J Infect Dis. 2013;208:1688-94.

9. Collins KA, Wang CY, Adams M, Mitchell H, Robinson GJ, Rampton M, et al. A Plasmodium vivax experimental human infection model for evaluating the efficacy of interventions. J Clin Investig. 2020;1 (130):2920-7.

10. Collins KA, Ruckle T, Elliott S, Marquart L, Ballard E, Chalon S, et al. DSM265 at 400 milligrams clears asexual stage parasites but not mature gametocytes from the blood of healthy subjects experimentally infected with Plasmodium falciparum. Antimicrob Agents Chemother. 2019;63.

11. Ward D. Conventional apheresis therapies: a review. J Clin Apher. 2011;26:230-8.

12. World Health Organization, Communicable Diseases Cluster. Severe falciparum malaria. Trans R Soc Trop Med Hyg. 2000;94 Suppl 1: S1-90.

13. Dondorp A, Nosten F, Stepniewska K, Day N, White N, South East Asian Quinine Artesunate Malaria Trial Group. Artesunate versus quinine for treatment of severe falciparum malaria: a randomised trial. Lancet. 2005;366:717-25

14. Dondorp AM, Fanello Cl, Hendriksen IC, Gomes E, Seni A, Chhaganlal KD, et al. Artesunate versus quinine in the treatment of severe falciparum malaria in African children (AQUAMAT): an open-label, randomised trial. Lancet. 2010;376:1647-57.

15. Ponnudurai T, Leeuwenberg AD, Meuwissen JH. Chloroquine sensitivity of isolates of Plasmodium falciparum adapted to in vitro culture. Trop Geogr Med. 1981;33:50-4.

16. Rockett RJ, Tozer SJ, Peatey C, Bialasiewicz S, Whiley DM, Nissen MD, et al. A real-time, quantitative PCR method using hydrolysis probes for the monitoring of Plasmodium falciparum load in experimentally infected human volunteers. Malar J. 2011;10:48.

17. U.S. Department of Health and Human Services, 2010. Common Terminology Criteria for Adverse Events (CTCAE) version 4.03 Institute National institutes of health National Cancer Institute. Available at: https://www. eortc.be/services/doc/ctc/CTCAE_4.03_2010-06-14_QuickReference_5x7. pdf. Accessed 06 April 2018. 
18. Cheng Q, Lawrence G, Reed C, Stowers A, Ranford-Cartwright L, Creasey $A$, et al. Measurement of Plasmodium falciparum growth rates in vivo: a test of malaria vaccines. Am J Trop Med Hyg. 1997;57:495-500.

19. Safeukui I, Millet P, Boucher S, Melinard L, Fregeville F, Receveur MC, et al. Evaluation of FRET real-time PCR assay for rapid detection and differentiation of Plasmodium species in returning travellers and migrants. Malar J. 2008;7:70.

20. Pasay CJ, Rockett R, Sekuloski S, Griffin P, Marquart L, Peatey C, et al. Piperaquine monotherapy of drug-susceptible Plasmodium falciparum infection results in rapid clearance of parasitemia but is followed by the appearance of gametocytemia. J Infect Dis. 2016;214:105-13.

21. Tsuboi T, Kaslow DC, Gozar MM, Tachibana M, Cao YM, Torii M. Sequence polymorphism in two novel Plasmodium vivax ookinete surface proteins, Pvs25 and Pvs28, that are malaria transmission-blocking vaccine candidates. Mol Med. 1998;4:772-82.

22. Collins KA, Wang CY, Adams M, Mitchell H, Rampton M, Elliott $S$, et al. A controlled human malaria infection model enabling evaluation of transmission-blocking interventions. J Clin Investig. 2018;128:1551-62.

23. Wang CY, McCarthy JS, Stone WJ, Bousema T, Collins KA, Bialasiewicz S. Assessing Plasmodium falciparum transmission in mosquito-feeding assays using quantitative PCR. Malar J. 2018;17:249.

24. Verhave JP, Faas B, Tchuinkam T, Arens T, Robert V. Detection of Plasmodium falciparum gametocytes by quantitative buffy coat analysis. Poster session presented at: $6^{\text {th }}$ Malaria Meeting of BSP; 1994 Sept 10-21; Liverpool
25. Wampfler R, Mwingira F, Javati S, Robinson L, Betuela I, Siba P, et al. Strategies for detection of Plasmodium species gametocytes. PLOS ONE. 2013;8:e76316.

26. Kim CC, Wilson EB, DeRisi JL. Improved methods for magnetic purification of malaria parasites and haemozoin. Malar J. 2010;9:17.

27. Clarke $M$, Ferreira $M$, Duraisingh $M$. The greater osmotic stability of reticulocytes is lost upon Plasmodium vivax infection. Poster session presented at: 7th International Conference on Plasmodium vivax research; 2019 Jun 26-28: Paris.

28. Ogwan'g RA, Mwangi JK, Githure J, Were JB, Roberts CR, Martin SK. Factors affecting exflagellation of in vitro-cultivated Plasmodium falciparum gametocytes. Am J Trop Med Hyg. 1993;49:25-9.

29. Billker O, Shaw MK, Margos G, Sinden RE. The roles of temperature, pH and mosquito factors as triggers of male and female gametogenesis of Plasmodium berghei in vitro. Parasitology. 1997;115:1-7.

30. Graumans W, Andolina C, Awandu SS, Grignard L, Lanke K, Bousema T. Plasmodium falciparum gametocyte enrichment in peripheral blood samples by magnetic fractionation: gametocyte yields and possibilities to reuse columns. Am J Trop Med Hyg. 2019;100:572-7.

\section{Publisher's Note}

Springer Nature remains neutral with regard to jurisdictional claims in published maps and institutional affiliations.
Ready to submit your research? Choose BMC and benefit from:

- fast, convenient online submission

- thorough peer review by experienced researchers in your field

- rapid publication on acceptance

- support for research data, including large and complex data types

- gold Open Access which fosters wider collaboration and increased citations

- maximum visibility for your research: over 100M website views per year

At BMC, research is always in progress.

Learn more biomedcentral.com/submissions 